\title{
Erratum to: Strahlentherapie und Onkologie, Volume 186 (No. 10)
}

Erratum to:

Guckenberger M, Ok S, Polat B, Sweeney RA, Flentje M. Toxicity after Intensity-Modulated, Image-Guided Radiotherapy for Prostate Cancer. Strahlenther Onkol 2010;186:535-43

(DOI 10.1007/s00066-010-2144-z)

Due to a technical error in the publishing process, several figures were inaccurately presented in Table 1. Please note the corrected data of "Tumor stage" and "Risk group."

Table 1. Patient and treatment characteristics. PO: prostate only; PSA: prostate-specific antigen; PTV: planning target volume; TURP: transurethral resection of the prostate; WP: whole pelvis.

Tabelle 1. Patienten- und Behandlungscharakteristika. PO: nur Prostata; PSA: prostataspezifisches Antigen; PTV: Planungszielvolumen; TURP: transurethrale Prostataresektion; WP: gesamtes Becken.

\begin{tabular}{|c|c|c|c|c|}
\hline & \multicolumn{2}{|c|}{$P 0(n=75)$} & \multicolumn{2}{|c|}{ WP $(n=25)$} \\
\hline Age (years), median (range) & \multicolumn{2}{|c|}{$73(54-80)$} & \multicolumn{2}{|c|}{$73(58-80)$} \\
\hline \multirow{3}{*}{$\begin{array}{l}\text { PSA level ( } \mathrm{ng} / \mathrm{ml}) \text {, median } \\
\text { (range) }\end{array}$} & \multicolumn{2}{|c|}{$7.7(1.5-32)$} & \multicolumn{2}{|c|}{$24.8(3.2-118)$} \\
\hline & \multicolumn{2}{|c|}{ Patients } & \multicolumn{2}{|c|}{ Patients } \\
\hline & $\mathrm{n}$ & $\%$ & $\mathrm{n}$ & $\%$ \\
\hline \multicolumn{5}{|l|}{ Gleason Score } \\
\hline - 2-6 & 38 & 50.7 & 5 & 20.0 \\
\hline - 7 & 33 & 44.0 & 9 & 36.0 \\
\hline - $8-9$ & 3 & 4.0 & 10 & 40.0 \\
\hline - Unknown & 1 & 1.3 & 1 & 4.0 \\
\hline \multicolumn{5}{|l|}{ Tumor stage } \\
\hline - $\mathrm{T} 1$ & 46 & 61.3 & 6 & 24.0 \\
\hline - $\mathrm{T} 2$ & 27 & 36.0 & 11 & 44.0 \\
\hline - $\mathrm{T} 3$ & 2 & 2.7 & 7 & 28.0 \\
\hline - $\mathrm{T} 4$ & 0 & 0.0 & 1 & 4.0 \\
\hline Androgen suppression & 23 & 30.7 & 16 & 64.0 \\
\hline TURP & 19 & 25.3 & 6 & 24.0 \\
\hline \multicolumn{5}{|l|}{ Risk group } \\
\hline - Low-risk & 29 & 38.7 & 0 & 0.0 \\
\hline - Intermediate-risk & 29 & 38.7 & 3 & 12.0 \\
\hline - High-risk & 17 & 22.7 & 22 & 88.0 \\
\hline \multicolumn{5}{|l|}{$\begin{array}{l}\text { Total dose (mean dose } \\
\text { PTV-2) }\end{array}$} \\
\hline - $73.91 \mathrm{~Gy}$ & 24 & 32.0 & 2 & 8.0 \\
\hline - $76.23 \mathrm{~Gy}$ & 51 & 68.0 & 23 & 92.0 \\
\hline
\end{tabular}

Strahlenther Onkol 2010;186:705

DOI 10.1007/s00066-010-7144-5

Published Online: November 29, 2010 